Article

\title{
Integral Characterizations for Uniform Stability with Growth Rates in Banach Spaces
}

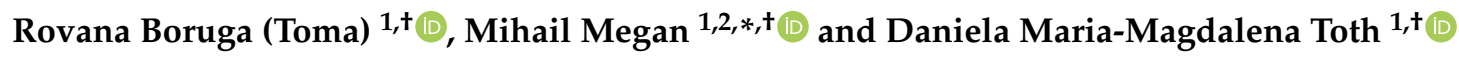 \\ 1 Department of Mathematics, Faculty of Mathematics and Computer Science, West University of Timişoara, \\ 300223 Timişoara, Romania; rovanaboruga@gmail.com (R.B.); dannielatoth@gmail.com (D.M.-M.T.) \\ 2 Academy of Romanian Scientists, 050094 Bucharest, Romania \\ * Correspondence: mihail.megan@e-uvt.ro \\ + These authors contributed equally to this work.
}

check for updates

Citation: Boruga (Toma), R.;

Megan, M.; Toth, D.M.-M. Integral

Characterizations for Uniform Stability with Growth Rates in Banach Spaces. Axioms 2021, 10, 235. https://doi.org/10.3390/

axioms10030235

Academic Editor: Davor Dragicevic

Received: 6 July 2021

Accepted: 14 September 2021

Published: 21 September 2021

Publisher's Note: MDPI stays neutral with regard to jurisdictional claims in published maps and institutional affiliations.

Copyright: (c) 2021 by the authors. Licensee MDPI, Basel, Switzerland. This article is an open access article distributed under the terms and conditions of the Creative Commons Attribution (CC BY) license (https:// creativecommons.org/licenses/by/ $4.0 /)$.

\begin{abstract}
The aim of this paper is to present some integral characterizations for the concept of uniform stability with growth rates in Banach spaces. In this sense, we prove necessary and sufficient conditions (of Barbashin and Datko type) for an evolution operator to be uniform h- stable. As particular cases of this notion, we obtain four characterizations for uniform exponential stability and two characterizations for uniform polynomial stability.
\end{abstract}

Keywords: evolution operator; uniform h-stability; Barbashin type characterizations; Datko type characterizations

MSC: 34D05; 34D20; 40C10

\section{Introduction}

In the last decades, the stability theory for dynamical systems in Banach spaces has been intensively studied. In this sense, we recall the monographs of E. A. Barbashin [1], J. L. Daleckii and M. G. Krein [2], L. Barreira and C. Valls [3]. Among the most important stability concepts studied in the literature, we mention the properties of uniform exponential stability and uniform polynomial stability.

The interesting part of a polynomial behavior lies in the fact that it is a weaker requirement than the corresponding exponential behavior. In other words, we can state that an evolution operator that is exponentially stable is also polynomially stable, but, in general, the converse implication is not true.

There are two remarkable results in the theory of uniform exponential stability due to E. A. Barbashin [1] and R. Datko [4]. After the seminal research of Barbashin and Datko, there has been a large number of papers devoted to this subject [5-12]. Generalizations of Barbashin and Datko's results for the case of the polynomial behaviors are given in [13-16].

In the present paper, we approach the concept of uniform stability with growth rates, where by the growth rate, we understand a bijective and nondecreasing application $h: \mathbb{R}_{+} \rightarrow[1, \infty)$. This concept was firstly introduced by M. Pinto [17] in his work in 1984 with the intention of obtaining results about stability for a weakly stable system under some perturbations. Furthermore, it was studied in the papers [18,19].

The purpose of this paper is to obtain some generalizations of the classical results due to Barbashin and Datko by giving some integral characterizations of the general concept of uniform stability with growth rates for evolution operators in Banach spaces. In this sense, we prove five necessary and sufficient conditions for the uniform $h$ - stability behavior: two conditions of Barbshin-type and three conditions of Datko-type. From these theorems, it follows as particular cases, four characterizations for uniform exponential stability and two characterizations for uniform polynomial stability. 
We remark that the sets of growth rates considered in this paper are different from those studied in [19]. Moreover, the results obtained for the exponential and polynomial cases are also distinct from those presented in the papers [6-15].

\section{Preliminaries}

Let $X$ be a real or complex Banach space and $X^{*}$ its dual space. We denote by $\mathcal{B}(X)$ the Banach algebra of all bounded linear operators acting on $X$. We denote by $I$ the identity operator and the norms on $X, X^{*}$ and on $\mathcal{B}(X)$ will be denoted by $\|$.$\| . By \Delta$ and $T$ we will denote the following sets

$$
\Delta=\left\{(t, s) \in \mathbb{R}_{+}^{2}: t \geq s\right\}, \quad T=\left\{\left(t, s, t_{0}\right) \in \mathbb{R}_{+}^{3}: t \geq s \geq t_{0}\right\} .
$$

Definition 1. We say that an application $\Phi: \Delta \rightarrow \mathcal{B}(X)$ is called evolution operator on $X$ if the following relations are satisfied:

$\left(e_{1}\right) \Phi(t, t)=I$, for all $t \geq 0$.

$\left(e_{2}\right) \Phi(t, s) \Phi\left(s, t_{0}\right)=\Phi\left(t, t_{0}\right)$, for all $\left(t, s, t_{0}\right) \in T$.

In addition,

$\left(e_{3}\right)$ if for all $(s, x) \in \mathbb{R}_{+} \times X$, the mapping $t \mapsto\|U(t, s) x\|$ is measurable on $[s, \infty)$, then the evolution operator $\Phi: \Delta \rightarrow \mathcal{B}(X)$ is said to be strongly measurable.

$\left(e_{4}\right)$ if for all $\left(s, x^{*}\right) \in \mathbb{R}_{+} \times X^{*}$, the mapping $t \mapsto\left\|U(t, s)^{*} x^{*}\right\|$ is measurable on $[0, t)$, then the evolution operator $\Phi: \Delta \rightarrow \mathcal{B}(X)$ is said to be $*$-strongly measurable.

In what follows, we consider $h: \mathbb{R}_{+} \rightarrow[1, \infty)$ a growth rate, which means that it is nondecreasing and bijective.

Definition 2. We say that the evolution operator $\Phi: \Delta \rightarrow \mathcal{B}(X)$ is called uniformly h-stable (u.h.s.) if there exist two constants $N>1$ and $v \in(0,1)$ such that

$$
h(t)^{v}\|\Phi(t, s) x\| \leq N h(s)^{v}\|x\|, \text { for all }(t, s, x) \in \Delta \times X .
$$

As particular cases, we have that

- $\quad$ if $h(s)=e^{s}$ then, the uniform exponential stability (u.e.s.) concept is obtained.

- if $h(s)=s+1$ then, the uniform polynomial stability (u.p.s.) concept is obtained.

Remark 1. $\Phi: \Delta \rightarrow \mathcal{B}(X)$ is an evolution operator, which is uniformly h-stable if and only if there exist two constants $N>1$ and $v \in(0,1)$ such that

$$
h(t)^{v}\left\|\Phi\left(t, t_{0}\right) x_{0}\right\| \leq N h(s)^{v}\left\|\Phi\left(s, t_{0}\right) x_{0}\right\|, \text { for all }\left(t, s, t_{0}, x_{0}\right) \in T \times X .
$$

Definition 3. We say that the evolution operator $\Phi: \Delta \rightarrow \mathcal{B}(X)$ has uniform h-growth (u.h.g.) if there exist two constants $M>1$ and $\omega>0$ such that

$$
h(s)^{\omega}\|\Phi(t, s) x\| \leq M h(t)^{\omega}\|x\|, \text { for all }(t, s, x) \in \Delta \times X .
$$

As particular cases, we have that

- $\quad$ if $h(t)=e^{t}$ the evolution operator has uniform exponential growth (u.e.g.).

- if $h(t)=t+1$ the evolution operator has uniform polynomial growth (u.p.g.).

Remark 2. $\Phi: \Delta \rightarrow \mathcal{B}(X)$ is an evolution operator, which has uniform h-growth if and only if there exist two constants $M>1$ and $\omega>0$ such that

$$
h(s)^{\omega}\left\|\Phi\left(t, t_{0}\right) x_{0}\right\| \leq M h(t)^{\omega}\left\|\Phi\left(s, t_{0}\right) x_{0}\right\|, \text { for all }\left(t, s, t_{0}, x_{0}\right) \in T \times X .
$$


Remark 3. If $\Phi: \Delta \rightarrow \mathcal{B}(X)$ is an evolution operator that is u.h.s., then it has u.h.g. The converse implication is not true in general. An example in this sense can be found in [18].

Remark 4. The next diagram provides the connections between the exponential and the polynomial concepts.

$$
\begin{array}{ccc}
\text { u.e.s. } & \Rightarrow & \text { u.e.g. } \\
\Downarrow & & \Uparrow \\
\text { u.p.s. } & \Rightarrow & \text { u.p.g. }
\end{array}
$$

Remark 5. The converse implications from the above diagram are not true. Indeed, if we consider the evolution operator

$$
\Phi: \Delta \rightarrow \mathcal{B}(\mathbb{R}), \Phi(t, s) x=\frac{\varphi(t)}{\varphi(s)} x
$$

then for

1. $\varphi(t)=\frac{1}{t^{2}+1}$ we have that $\Phi$ is uniformly polynomially stable and it is not uniformly exponentially stable.

2. $\varphi(t)=t+1$ we have that $\Phi$ has uniform polynomial growth and it is not uniformly polynomially stable.

3. $\varphi(t)=e^{t}$ we have that $\Phi$ has uniform exponential growth and it is not uniformly exponentially stable and it does not have uniform polynomial growth.

\section{Barbashin Type Criteria for Uniform $h$-Stability}

In this section, we will consider

- $\quad \mathcal{H}$ to be the set of all functions $h: \mathbb{R}_{+} \rightarrow[1, \infty)$ with the property that there exists $H>1$ such that $h(t+1) \leq H h(t)$, for all $t \geq 0$.

- $\quad \mathcal{H}_{1}$ to be the set of all functions $h: \mathbb{R}_{+} \rightarrow[1, \infty)$ with the property that there exists $H_{1}>1$ such that $h(h(t)) \leq H_{1} h(t)$, for all $t \geq 0$.

- $\quad \mathcal{H}_{B}^{1}$ to be the set of all functions $h: \mathbb{R}_{+} \rightarrow[1, \infty)$ with the property that for all $\alpha \in(0,1)$, there exists $H_{1}>1$ such that $\int_{0}^{t} h(s)^{\alpha-1} d s \leq H_{1} h(t)^{\alpha}$, for all $t \geq 0$.

- $\quad \mathcal{H}_{B}^{2}$ to be the set of all functions $h: \mathbb{R}_{+} \rightarrow[1, \infty)$ with the property that for all $\alpha \in(0,1)$, there exists $H_{2}>1$ such that $\int_{0}^{t} h(s)^{\alpha} d s \leq H_{2} h(t)^{\alpha}$, for all $t \geq 0$.

Remark 6. If e is an exponential function, then

$$
e \in \mathcal{H} \cap \mathcal{H}_{B}^{2} \subset \mathcal{H} \cap \mathcal{H}_{B}^{1} .
$$

Remark 7. If $p$ is a polynomial function, then

$$
p \in\left(\mathcal{H} \cap \mathcal{H}_{1}\right) \cup\left(\mathcal{H}_{B}^{1} \backslash \mathcal{H}_{B}^{2}\right)
$$

The following result is a characterization theorem of Barbashin-type for the uniform h-stability concept.

Theorem 1. Let $h \in \mathcal{H}_{1} \cap \mathcal{H}_{B}^{1}$ and $\Phi: \Delta \rightarrow \mathcal{B}(X)$ a *-strongly measurable evolution operator, which has uniform $h$-growth. Then, $\Phi$ is uniformly $h$ - stable if and only if there exist $B>1$ and $b \in(0,1)$ such that

$$
\int_{0}^{t} \frac{\left\|\Phi(t, s)^{*} x^{*}\right\|}{h(s)^{b+1}} d s \leq \frac{B\left\|x^{*}\right\|}{h(t)^{b}}
$$


for all $\left(t, x^{*}\right) \in \mathbb{R}_{+} \times X^{*}$.

Proof. Necessity. If we suppose that $\Phi$ is u.h.s., then we have that there exist two constants $N>1$ and $v \in(0,1)$ such that for $b \in(0, v)$ we have

$$
\begin{aligned}
\int_{0}^{t} \frac{\left\|\Phi(t, s)^{*} x^{*}\right\|}{h(s)^{b+1}} d s & \leq N \int_{0}^{t}\left(\frac{h(s)}{h(t)}\right)^{v} \frac{\left\|x^{*}\right\|}{h(s)^{b+1}} d s=\frac{N\left\|x^{*}\right\|}{h(t)^{v}} \int_{0}^{t} h(s)^{v-b-1} d s \leq \\
& \leq \frac{N\left\|x^{*}\right\|}{h(t)^{v}} \cdot H_{1} \cdot h(t)^{v-b}=\frac{N H_{1}\left\|x^{*}\right\|}{h(t)^{b}} \leq \frac{B\left\|x^{*}\right\|}{h(t)^{b}},
\end{aligned}
$$

where $B=\mathrm{NH}_{1}$.

Sufficiency. If $t>h(s)$ we have

$$
\begin{aligned}
& \frac{\left\|\Phi(t, s)^{*} x^{*}\right\|}{h(s)^{b}}=\frac{2}{h(s)} \int_{\frac{h(s)}{2}}^{h(s)} \frac{\left\|\Phi(t, s)^{*} x^{*}\right\|}{h(s)^{b}} d \tau=\frac{2}{h(s)} \int_{\frac{h(s)}{2}}^{h(s)} \frac{\Phi(\tau, s)^{*} \Phi(t, \tau)^{*} x^{*}}{h(s)^{b}} d \tau \leq \\
& \leq \frac{2 M}{h(s)} \int_{\frac{h(s)}{2}}^{h(s)} \frac{\left(\frac{h(\tau)}{h(s)}\right)^{\omega}\left\|\Phi(t, \tau)^{*} x^{*}\right\|}{h(s)^{b}} d \tau=2 M \int_{\frac{h(s)}{2}}^{h(s)}\left(\frac{h(\tau)}{h(s)}\right)^{\omega+b+1} \cdot \frac{\left\|\Phi(t, \tau)^{*} x^{*}\right\|}{h(\tau)^{b+1}} d \tau \leq \\
& \leq 2 M \int_{\frac{h(s)}{2}}^{h(s)}\left(\frac{h(h(s))}{h(s)}\right)^{\omega+b+1} \cdot \frac{\left\|\Phi(t, \tau)^{*} x^{*}\right\|}{h(\tau)^{b+1}} d \tau \leq 2 M H_{1}^{\omega+b+1} \int_{0}^{t} \frac{\left\|\Phi(t, \tau)^{*} x^{*}\right\|}{h(\tau)^{b+1}} d \tau \leq \\
& \leq 2 M H_{1}^{\omega+b+1} \cdot \frac{B\left\|x^{*}\right\|}{h(t)^{b}} .
\end{aligned}
$$

Thus, we obtain

$$
\left\|\Phi(t, s)^{*} x^{*}\right\| \leq 2 M H_{1}^{\omega+b+1} B\left(\frac{h(s)}{h(t)}\right)^{b}\left\|x^{*}\right\|, \text { for all } t>h(s) .
$$

If $t \in[s, h(s)]$ we have

$$
\begin{aligned}
h(t)^{b}\left\|\Phi(t, s)^{*} x^{*}\right\| & \leq M h(t)^{b} \cdot\left(\frac{h(t)}{h(s)}\right)^{\omega}\left\|x^{*}\right\|=M\left(\frac{h(t)}{h(s)}\right)^{\omega+b} h(s)^{b}\left\|x^{*}\right\| \leq \\
& \leq M\left(\frac{h(h(s))}{h(s)}\right)^{\omega+b} h(s)^{b}\left\|x^{*}\right\| \leq 2 M H_{1}^{\omega+b+1} B h(s)^{b}\left\|x^{*}\right\| .
\end{aligned}
$$

Hence,

$$
\left\|\Phi(t, s)^{*} x^{*}\right\| \leq N\left(\frac{h(s)}{h(t)}\right)^{b}\left\|x^{*}\right\|, \text { for all } t \in[s, h(s)],
$$

where $N=2 M H_{1}^{b+\omega+1} B$.

From (2) and (3), it follows that the relation (1) states for all $t \geq s \geq 0$, which means that $\Phi$ is u.h.s. and the theorem is proved.

As an immediate consequence of the theorem above, we obtain a version of Barbashin's theorem for the case of the uniform polynomial stability concept, given by: 
Corollary 1. If $\Phi: \Delta \rightarrow \mathcal{B}(X)$ is a $*$-strongly measurable evolution operator, which has uniform polynomial growth, then it is uniformly polynomially stable if and only if there exist two constants $B>1$ and $b \in(0,1)$ such that

$$
\int_{0}^{t} \frac{\left\|\Phi(t, s)^{*} x^{*}\right\|}{(s+1)^{b+1}} d s \leq \frac{B\left\|x^{*}\right\|}{(t+1)^{b}}, \text { for all }\left(t, x^{*}\right) \in \mathbb{R}_{+} \times X^{*}
$$

Proof. It follows from Theorem 1, taking $h(s)=s+1$.

The next theorem presents another characterization of Barbashin- type for uniform $h$-stability.

Theorem 2. Let $h \in \mathcal{H} \cap \mathcal{H}_{B}^{2}$ and $\Phi: \Delta \rightarrow \mathcal{B}(X) a *$-strongly measurable evolution operator, which has uniform h-growth. Then, $\Phi$ is uniformly $h$ - stable if and only if there exist $B>1$ and $b \in(0,1)$ such that

$$
\int_{0}^{t} \frac{\left\|\Phi(t, s)^{*} x^{*}\right\|}{h(s)^{b}} d s \leq \frac{B\left\|x^{*}\right\|}{h(t)^{b}}
$$

for all $\left(t, x^{*}\right) \in \mathbb{R}_{+} \times X^{*}$.

Proof. Necessity. We suppose that $\Phi$ is u.h.s. Then, there exist two constants $N>1$ and $v \in(0,1)$ such that for $b \in(0, v)$ we have

$$
\begin{aligned}
& \int_{0}^{t} \frac{\left\|\Phi(t, s)^{*} x^{*}\right\|}{h(s)^{b}} d s \leq N\left\|x^{*}\right\| \int_{0}^{t}\left(\frac{h(s)}{h(t)}\right)^{v} \cdot \frac{h(t)^{b}}{h(s)^{b} \cdot h(t)^{b}} d s= \\
& =N \frac{h(t)^{b-v}}{h(t)^{b}}\left\|x^{*}\right\| \int_{0}^{t} \frac{1}{h(s)^{b-v}} d s \leq N \frac{h(t)^{b-v}}{h(t)^{b}}\left\|x^{*}\right\| \cdot \frac{H_{2}}{h(t)^{b-v}} \leq \frac{B\left\|x^{*}\right\|}{h(t)^{b}},
\end{aligned}
$$

where $B=1+\mathrm{NH}_{2}$.

Sufficiency. If $t>s+1$ we have

$$
\begin{aligned}
h(t)^{b}\left\|\Phi(t, s)^{*} x^{*}\right\| & =\int_{s}^{s+1} h(t)^{b}\left\|\Phi(\tau, s)^{*} \Phi(t, \tau)^{*} x^{*}\right\| d \tau \leq \\
& \leq M h(t)^{b} \int_{s}^{s+1}\left(\frac{h(\tau)}{h(s)}\right)^{\omega}\left\|\Phi(t, \tau)^{*} x^{*}\right\| d \tau \cdot \frac{h(\tau)^{b}}{h(\tau)^{b}} \leq \\
& \leq M h(t)^{b} \int_{s}^{s+1} \frac{h(\tau)^{b+\omega}}{h(s)^{\omega} h(\tau)^{b}}\left\|\Phi(t, \tau)^{*} x^{*}\right\| d \tau \leq \\
& \leq M h(t)^{b} \int_{s}^{s+1}\left(\frac{h(s+1)}{h(s)}\right)^{b+\omega} \cdot h(s)^{b} \cdot \frac{\left\|\Phi(t, \tau)^{*} x^{*}\right\|}{h(\tau)^{b}} d \tau \leq \\
& \leq M H^{b+\omega} h(t)^{b} \cdot h(s)^{b} \int_{0}^{t} \frac{\left\|\Phi(t, \tau)^{*} x^{*}\right\|}{h(\tau)^{b}} d \tau \leq M B H^{b+\omega} \cdot h(s)^{b}\left\|x^{*}\right\| .
\end{aligned}
$$

Thus, we obtain

$$
\left\|\Phi(t, s)^{*} x^{*}\right\| \leq M B H^{b+\omega}\left(\frac{h(s)}{h(t)}\right)^{b}\left\|x^{*}\right\|, \text { for all } t>s+1 .
$$


If $t \in[s, s+1]$, we apply the growth property and we obtain

$$
\begin{aligned}
h(t)^{b}\left\|\Phi(t, s)^{*} x^{*}\right\| & \leq M h(t)^{b}\left(\frac{h(t)}{h(s)}\right)\left\|x^{*}\right\|=M\left(\frac{h(t)}{h(s)}\right)^{b+\omega} h(s)^{b}\left\|x^{*}\right\| \leq \\
& \leq M\left(\frac{h(s+1)}{h(s)}\right)^{b+\omega} h(s)^{b}\left\|x^{*}\right\| \leq M H^{b+\omega} h(s)^{b}\left\|x^{*}\right\| .
\end{aligned}
$$

Therefore, we have

$$
\left\|\Phi(t, s)^{*} x^{*}\right\| \leq M H^{b+\omega}\left(\frac{h(s)}{h(t)}\right)^{b}\left\|x^{*}\right\|, \text { for all } t \in[s, s+1] .
$$

In conclusion, from relations (5) and (6), it follows that

$$
\left\|\Phi(t, s)^{*} x^{*}\right\| \leq N\left(\frac{h(s)}{h(t)}\right)^{b}\left\|x^{*}\right\|, \text { for all } t \geq s \geq 0
$$

where $N=M B H^{b+\omega}$, which means that $\Phi$ is u.h.s.

As an immediate consequence of the theorem above, we obtain a version of Barbashin's theorem for the case of the uniform exponential stability concept, given by:

Corollary 2. If $\Phi: \Delta \rightarrow \mathcal{B}(X)$ is a $*$-strongly measurable evolution operator that has uniform exponential growth, then it is uniformly exponentially stable if and only if there exist two constants $B>1$ and $b \in(0,1)$ such that

$$
\int_{0}^{t} e^{-b s}\left\|\Phi(t, s)^{*} x^{*}\right\| d s \leq B e^{-b t}\left\|x^{*}\right\|, \text { for all }\left(t, x^{*}\right) \in \mathbb{R}_{+} \times X^{*} .
$$

Proof. It follows from Theorem 2, taking $h(s)=e^{s}$.

Remark 8. Another proof of the Corollary 2 can be found in [5] for the case $b=0$.

\section{Datko Type Characterizations for Uniform $h$-Stability}

In what follows, let us consider

- $\quad \mathcal{H}_{0}$ to be the set of all functions $h: \mathbb{R}_{+} \rightarrow[1, \infty)$ with the property that $h(t) \geq t+1$, for all $t \geq 0$.

- $\quad \mathcal{H}$ to be the set of all functions $h: \mathbb{R}_{+} \rightarrow[1, \infty)$ with the property that there exists $H>1$ such that $h(t+1) \leq H h(t)$, for all $t \geq 0$.

- $\quad \mathcal{H}_{D}^{1}$ to be the set of all functions $h: \mathbb{R}_{+} \rightarrow[1, \infty)$ with the property that for all $\alpha<0$, there exists $H_{1}>1$ such that $\int_{0}^{t} h(s)^{\alpha-1} d s \leq H_{1} h(t)^{\alpha}$, for all $s \geq 0$.

- $\mathcal{H}_{D}^{2}$ to be the set of all functions $h: \mathbb{R}_{+} \rightarrow[1, \infty)$ with the property that for all $\alpha<0$, there exists $H_{2}>1$ such that $\int_{0}^{t} h(s)^{\alpha} d s \leq H_{2} h(t)^{\alpha}$, for all $s \geq 0$.

- $\quad \mathcal{H}_{D}^{3}$ to be the set of all functions $h: \mathbb{R}_{+} \rightarrow[1, \infty)$ with the property that for all $\alpha>0$, there exists $H_{3}>1$ such that $\int_{0}^{t} h(s)^{\alpha} d s \leq H_{3} h(t)^{\alpha}$, for all $t \geq 0$.

Remark 9. If $e$ is an exponential function and $p$ is a polynomial function, then

- $\quad e, p \in \mathcal{H} \cap \mathcal{H}_{0}$ 
- $\quad e \in \mathcal{H}_{D}^{2} \cap \mathcal{H}_{D}^{3} \subset \mathcal{H}_{D}^{1} \cap \mathcal{H}_{D}^{3}$

- $\quad p \in \mathcal{H}_{D}^{1} \backslash\left(\mathcal{H}_{D}^{2} \cup \mathcal{H}_{D}^{3}\right)$

In what follows, we give a characterization theorem of Datko type for the uniform $h$-stability of an evolution operator.

Theorem 3. Let $h \in \mathcal{H}_{0} \cap \mathcal{H}_{D}^{1}$ and $\Phi: \Delta \rightarrow \mathcal{B}(X)$ be a strongly measurable evolution operator that has uniform $h$-growth. Then, $\Phi$ is uniformly $h$ - stable if and only if there exist two constants $D>1$ and $d \in(0,1)$ such that

$$
\int_{s}^{\infty} h(t)^{d-1}\|\Phi(t, s) x\| d t \leq D h(s)^{d}\|x\|,
$$

for all $(s, x) \in \mathbb{R}_{+} \times X$.

Proof. Necessity. We suppose that $\Phi$ is u.h.s. Then, it follows that there exist $N>1$ and $v \in(0,1)$ such that for $d \in(0, v)$ we have

$$
\begin{aligned}
& \int_{s}^{\infty} h(t)^{d-1}\|\Phi(t, s) x\| d t \leq N \int_{s}^{\infty} h(t)^{d-1}\left(\frac{h(s)}{h(t)}\right)^{v}\|x\| d t \leq \\
& \leq N\|x\| h(s)^{v} \int_{s}^{\infty} h(t)^{d-v-1} d t \leq N H_{1} h(s)^{v} h(s)^{d-v}\|x\| \leq D h(s)^{d}\|x\|,
\end{aligned}
$$

where $D=1+\mathrm{NH}_{1}$.

Sufficiency. If $(t, s) \in \Delta, h(t)>2 s$, we have

$$
\begin{aligned}
h(t)^{d}\|\Phi(t, s) x\| & =\frac{2}{h(t)} \int_{\frac{h(t)}{2}}^{h(t)} h(t)^{d}\|\Phi(t, \tau) \Phi(\tau, s) x\| d \tau \leq \\
& \leq \frac{2 M}{h(t)} \int_{\frac{h(t)}{2}}^{h(t)} h(t)^{d}\left(\frac{h(t)}{h(\tau)}\right)^{\omega}\|\Phi(\tau, s) x\| d \tau \leq \\
& \leq 2^{\omega+1} \cdot M \int_{\frac{h(t)}{2}}^{h(t)}\left(\frac{h(t)}{h(\tau)}\right)^{d-1} h(\tau)^{d-1}\|\Phi(\tau, s) x\| d \tau \leq \\
& \leq 2^{\omega+d} \cdot M \cdot \int_{s}^{\infty} h(\tau)^{d-1}\|\Phi(\tau, s) x\| d \tau \leq 2^{\omega+d} \cdot M \cdot D h(s)^{d}\|x\| .
\end{aligned}
$$

Thus, we obtain

$$
h(t)^{d}\|\Phi(t, s) x\| \leq 2^{\omega+d} \cdot M \cdot D \cdot h(s)^{d}\|x\|, \text { for all }(t, s) \in \Delta, h(t)>2 s .
$$
obtain

If $(t, s) \in \Delta, h(t)<2 s$, we use the growth property of the evolution operator and we

$$
\begin{aligned}
h(t)^{d}\|\Phi(t, s) x\| & \leq M h(t)^{d}\left(\frac{h(t)}{h(s)}\right)^{\omega}\|x\|=M\left(\frac{h(t)}{h(s)}\right)^{d+\omega} h(s)^{d}\|x\| \leq \\
& \leq M \cdot 4^{d+\omega} h(s)^{d}\|x\| .
\end{aligned}
$$


It follows that

$$
h(t)^{d}\|\Phi(t, s) x\| \leq 4^{\omega+d} \cdot M \cdot h(s)^{d}\|x\|, \text { for all }(t, s) \in \Delta, h(t)<2 s .
$$

From relations (8) and (9), we obtain that there exist $N=4^{d+\omega} M D>1$ and $v=$ $d \in(0,1)$ such that $(7)$ is satisfied for all pairs $(t, s) \in \Delta$, which means that the proof is completed.

As a consequence of the theorem presented above, we deduce the following corollaries, which are versions of Datko's theorem for the case of the uniform exponential stability and uniform polynomial stability concepts.

Corollary 3. If $\Phi: \Delta \rightarrow \mathcal{B}(X)$ is a strongly measurable evolution operator that has uniform exponential growth, then it is uniformly exponentially stable if and only if there exist two constants $D>1$ and $d \in(0,1)$ such that

$$
\int_{s}^{\infty} e^{(d-1) t}\|\Phi(t, s) x\| d t \leq D \cdot e^{d s}\|x\|
$$

for all $(s, x) \in \mathbb{R}_{+} \times X$.

Proof. It follows from Theorem 3, if we consider $h(t)=e^{t}$.

Corollary 4. If $\Phi: \Delta \rightarrow \mathcal{B}(X)$ is a strongly measurable evolution operator that has uniform polynomial growth, then it is uniformly polynomially stable if and only if there exist two constants $D>1$ and $d \in(0,1)$ such that

$$
\int_{s}^{\infty}(t+1)^{(d-1)}\|\Phi(t, s) x\| d t \leq D \cdot(s+1)^{d}\|x\|,
$$

for all $(s, x) \in \mathbb{R}_{+} \times X$.

Proof. It follows from Theorem 3, if we consider $h(t)=t+1$.

Another characterization due to Datko for the uniform $h$-stability concept is given by:

Theorem 4. Let $h \in \mathcal{H} \cap \mathcal{H}_{D}^{2}$ and $\Phi: \Delta \rightarrow \mathcal{B}(X)$ be a strongly measurable evolution operator that has uniform h-growth. Then, $\Phi$ is uniformly $h$ - stable if and only if there exist $D>1$ and $d \in(0,1)$ such that

$$
\int_{s}^{\infty} h(t)^{d}\|\Phi(t, s) x\| d t \leq D \cdot h(s)^{d}\|x\|,
$$

for all $(s, x) \in \mathbb{R}_{+} \times X$.

Proof. Necessity. We suppose that $\Phi$ is u.h.s. Let $d \in(0, v) \subset(0,1)$. Then, we have

$$
\begin{aligned}
& \int_{s}^{\infty} h(t)^{d}\|\Phi(t, s) x\| d t \leq N \int_{s}^{\infty} h(t)^{d}\left(\frac{h(s)}{h(t)}\right)^{v}\|x\| d t= \\
& =N\|x\| h(s)^{v} \int_{s}^{\infty} h(t)^{d-v} d t \leq N H_{2} h(s)^{v} h(s)^{d-v}\|x\|=D h(s)^{d}\|x\|,
\end{aligned}
$$

where $\mathrm{D}=\mathrm{N} \cdot \mathrm{H}_{2}$. 
Sufficiency. If $t \geq s+1$, we have

$$
\begin{aligned}
& h(t)^{d}\|\Phi(t, s) x\|=h(t)^{d} \int_{t-1}^{t}\|\Phi(t, \tau) \Phi(\tau, s) x\| d \tau \leq \\
& \leq M h(t)^{d} \int_{t-1}^{t}\left(\frac{h(t)}{h(\tau)}\right)^{\omega}\|\Phi(\tau, s) x\| d \tau= \\
& =M \int_{t-1}^{t}\left(\frac{h(t)}{h(\tau)}\right)^{d+\omega} \cdot h(\tau)^{d}\|\Phi(\tau, s) x\| d \tau \leq \\
& \leq M \int_{t-1}^{t}\left(\frac{h(t)}{h(t-1)}\right)^{d+\omega} \cdot h(\tau)^{d}\|\Phi(\tau, s) x\| d \tau \leq \\
& \leq M \cdot H^{d+\omega} \int_{s}^{\infty} h(\tau)^{d}\|\Phi(\tau, s) x\| d \tau \leq M \cdot D \cdot H^{d+\omega} \cdot h(s)^{d}\|x\| .
\end{aligned}
$$

Thus, we obtain

$$
h(t)^{d}\|\Phi(t, s) x\| \leq M \cdot D \cdot H^{d+\omega} \cdot h(s)^{d}\|x\|, \text { for all } t \geq s+1 .
$$

If $t \in[s, s+1]$, using the growth property we obtain

$$
\begin{aligned}
h(t)^{d}\|\Phi(t, s) x\| & \leq M h(t)^{d}\left(\frac{h(t)}{h(s)}\right)^{\omega}\|x\|=M\left(\frac{h(t)}{h(s)}\right)^{d+\omega} h(s)^{d}\|x\| \leq \\
& \leq M\left(\frac{h(s+1)}{h(s)}\right)^{d+\omega} h(s)^{d}\|x\| \leq M \cdot H^{d+\omega} h(s)^{d}\|x\| .
\end{aligned}
$$

It follows that

$$
h(t)^{d}\|\Phi(t, s) x\| \leq M \cdot H^{\omega+d} \cdot h(s)^{d}\|x\|, \text { for all } t \in[s, s+1] .
$$

From relations (11) and (12) we obtain that there exist $N=M D H^{d+\omega}>1$ and $v=d \in(0,1)$ such that $(10)$ is satisfied for all pairs $(t, s) \in \Delta$, which means that the proof is completed.

As an immediate consequence of the theorem above, we obtain a version of Datko's theorem for the case of the uniform exponential stability concept, given by:

Corollary 5. If $\Phi: \Delta \rightarrow \mathcal{B}(X)$ is a strongly measurable evolution operator that has uniform exponential growth, then it is uniformly exponentially stable if and only if there exist two constants $D>1$ and $d \in(0,1)$ such that

$$
\int_{s}^{\infty} e^{d t}\|\Phi(t, s) x\| d t \leq D e^{d s}\|x\|, \text { for all }(s, x) \in \mathbb{R}_{+} \times X .
$$

Proof. It follows from Theorem 4 , taking $h(t)=e^{t}$.

The next result gives an integral characterization of Datko type for the concept of uniform stability with growth rates. 
Theorem 5. Let $h \in \mathcal{H} \cap \mathcal{H}_{D}^{3}$ and $\Phi: \Delta \rightarrow \mathcal{B}(X)$ be a strongly measurable evolution operator that has uniform $h$-growth. Then, $\Phi$ is uniformly $h$ - stable if and only if there exist $D>1$ and $d \in(0,1)$ such that

$$
\int_{t_{0}}^{t} \frac{d s}{h(s)^{d}\left\|\Phi\left(s, t_{0}\right) x_{0}\right\|} \leq \frac{D}{h(t)^{d}\left\|\Phi\left(t, t_{0}\right) x_{0}\right\|},
$$

for all $\left(t, t_{0}, x_{0}\right) \in \Delta \times X$, with $\Phi\left(t, t_{0}\right) x_{0} \neq 0$.

Proof. Necessity. We suppose that $\Phi$ is u.h.s. Then, there exist two constants $N>1$ and $d \in(0,1)$ such that for $d \in(0, v)$ we have

$$
\begin{aligned}
& \int_{t_{0}}^{t} \frac{d s}{h(s)^{d}\left\|\Phi\left(s, t_{0}\right) x_{0}\right\|} \leq N \int_{t_{0}}^{t} \frac{1}{h(s)^{d}}\left(\frac{h(s)}{h(t)}\right)^{v} \cdot \frac{1}{\left\|\Phi\left(t, t_{0}\right) x_{0}\right\|} d s= \\
& =\frac{N}{h(t)^{v}\left\|\Phi\left(t, t_{0}\right) x_{0}\right\|} \int_{t_{0}}^{t} h(s)^{v-d} d s \leq \frac{N H_{3}}{h(t)^{v}\left\|\Phi\left(t, t_{0}\right) x_{0}\right\|} \cdot h(t)^{v-d}= \\
& =\frac{D}{h(t)^{d}\left\|\Phi\left(t, t_{0}\right) x_{0}\right\|},
\end{aligned}
$$

where $\mathrm{D}=\mathrm{N} \cdot \mathrm{H}_{3}$.

Sufficiency. If $t \geq s+1, \Phi\left(t, t_{0}\right) x_{0} \neq 0$, we have

$$
\begin{aligned}
\frac{1}{h(s)^{d}\left\|\Phi\left(s, t_{0}\right) x_{0}\right\|} & =\int_{s}^{s+1} \frac{d \tau}{h(s)^{d}\left\|\Phi\left(s, t_{0}\right) x_{0}\right\|} \leq \\
& \leq M \int_{s}^{s+1} \frac{1}{h(s)^{d}}\left(\frac{h(\tau)}{h(s)}\right)^{\omega} \cdot \frac{h(\tau)^{-d} h(\tau)^{d} d \tau}{\| \Phi\left(\tau, t_{0}\right) x_{0}}= \\
& =M \int_{s}^{s+1}\left(\frac{h(\tau)}{h(s)}\right)^{d+\omega} \cdot \frac{d \tau}{h(\tau)^{d}\left\|\Phi\left(\tau, t_{0}\right) x_{0}\right\|} \leq \\
& \leq M \int_{s}^{s+1}\left(\frac{h(s+1)}{h(s)}\right)^{d+\omega} \cdot \frac{d \tau}{h(\tau)^{d}\left\|\Phi\left(\tau, t_{0}\right) x_{0}\right\|} \leq \\
& \leq M \cdot H^{d+\omega} \int_{t_{0}}^{t} \frac{d \tau}{h(\tau)^{d}\left\|\Phi\left(\tau, t_{0}\right) x_{0}\right\|} \leq \\
& \leq D M H^{d+\omega} \cdot \frac{1}{h(t)^{d}\left\|\Phi\left(t, t_{0}\right) x_{0}\right\|} .
\end{aligned}
$$

Thus, we obtain

$$
h(t)^{d}\left\|\Phi\left(t, t_{0}\right) x_{0}\right\| \leq D M H^{d+\omega} \cdot h(s)^{d}\left\|\Phi\left(s, t_{0}\right) x_{0}\right\|, \text { for all } t \geq s+1 .
$$

If $t \in[s, s+1]$ and $\Phi\left(s, t_{0}\right) x_{0} \neq 0$, using the growth property, we obtain

$$
\begin{aligned}
\left\|\Phi\left(t, t_{0}\right) x_{0}\right\| & \leq M\left(\frac{h(t)}{h(s)}\right)^{\omega}\left\|\Phi\left(s, t_{0}\right) x_{0}\right\| \leq M\left(\frac{h(s+1)}{h(s)}\right)^{\omega}\left\|\Phi\left(s, t_{0}\right) x_{0}\right\| \leq \\
& \leq M H^{\omega}\left\|\Phi\left(s, t_{0}\right) x_{0}\right\| .
\end{aligned}
$$


It follows that

$$
\begin{aligned}
h(t)^{d}\left\|\Phi\left(t, t_{0}\right) x_{0}\right\| & \leq M H^{\omega} h(t)^{d}\left\|\Phi\left(s, t_{0}\right) x_{0}\right\| \leq M H^{\omega}\left(\frac{h(t)}{h(s)}\right)^{d} \cdot h(s)^{d}\left\|\Phi\left(s, t_{0}\right) x_{0}\right\| \leq \\
& \leq M H^{d+\omega} h(s)^{d}\left\|\Phi\left(s, t_{0}\right) x_{0}\right\| .
\end{aligned}
$$

Thus, we obtain

$$
h(t)^{d}\left\|\Phi\left(t, t_{0}\right) x_{0}\right\| \leq M H^{\omega+d} \cdot h(s)^{d}\left\|\Phi\left(s, t_{0}\right) x_{0}\right\|, \text { for all } t \in[s, s+1] .
$$

From relations (14) and (15), we obtain that there exist $N=M D H^{d+\omega}>1$ and $v=d \in(0,1)$ such that (13) is satisfied for all pairs $(t, s) \in \Delta$, which means that the proof is completed.

As an immediate consequence of the theorem above, we obtain another version of Datko's theorem for the case of the uniform exponential stability concept, given by:

Corollary 6. If $\Phi: \Delta \rightarrow \mathcal{B}(X)$ is a strongly measurable evolution operator that has uniform exponential growth, then it is uniformly exponentially stable if and only if there exist two constants $D>1$ and $d \in(0,1)$ such that

$$
\int_{t_{0}}^{t} \frac{d s}{e^{d s}\left\|\Phi\left(s, t_{0}\right) x_{0}\right\|} \leq \frac{D}{e^{d t}\left\|\Phi\left(t, t_{0}\right) x_{0}\right\|},
$$

for all $\left(t, t_{0}, x_{0}\right) \in \Delta \times X$, with $\Phi\left(t, t_{0}\right) x_{0} \neq 0$.

Proof. It is a particular case of Theorem 5 for $h(t)=e^{t}$.

\section{Conclusions}

The main purpose of the present paper is to give some generalizations of the classical results due to Barbashin [1] and Datko [4] about integral characterizations of uniform exponential stability of evolution operators in Banach spaces for the general case of uniform stability with growth rates.

More precisely, we prove two characterizations of Barbashin-type and three characterizations of Datko-type for uniform $h$-stability. As particular cases, we obtain four integral characterizations for uniform exponential stability and two necessary and sufficient conditions for uniform polynomial stability.

In the future, the authors would like to study the variants of these results in the nonuniform case and generalizations for the dichotomies and trichotomies behaviors.

Author Contributions: All authors contributed equally to this work. All authors have read and agreed to the published version of the manuscript.

Funding: This research received no external funding.

Institutional Review Board Statement: Not applicable.

Informed Consent Statement: Not applicable.

Data Availability Statement: Not applicable.

Acknowledgments: The authors would like to thank the reviewers for his/her constructive comments and suggestions.

Conflicts of Interest: The authors declare no conflict of interest. 


\section{References}

1. Barbashin, E.A. Introduction in the Theory of Stability; Izd. Nauka: Moscow, Russia, 1967.

2. Daleckii, L.; Krein, M.G. Stability of Solutions of Differential Equations in Banach Spaces; American Mathematical Society: Providence, RI, USA, 1974.

3. Barreira, L.; Valls, C. Stability of Nonautonomous Differential Equations; Lecture Notes in Mathematics; Springer: Berlin, Germany, 2008.

4. Datko, R. Uniform asymptotic stability of evolutionary processes in a Banach space. SIAM J. Math. Anal. 1972, 3, 428-445. [CrossRef]

5. Buşe, C.; Megan, M.; Prajea, M.; Preda, P. The strong variant of a Barbashin theorem on stability of solutions for nonautonomous differential equations in Banach spaces. Integral Equations Oper. Theory 2007, 59, 491-500. [CrossRef]

6. Hai, P.V. Two new approaches to Barbashin theorem. Dyn. Contin. Discret. Impuls. Syst. 2012, 19, 773-798.

7. Hai, P.V. A Generalization for Theorems of Datko and Barbashin Type. J. Funct. Spaces 2015, 2015, 517348. [CrossRef]

8. Hai, P.V. Discrete and continuous versions of Barbashin-type theorem of linear skew-evolution semiflows. Appl. Anal. 2011, 90, 1897-1907. [CrossRef]

9. Dragičević, D. Barbashin-type conditions for exponential stability of linear cocycles. Monatshefte FÜR Math. 2020, 192, 813-826. [CrossRef]

10. Dragičević, D. A version of a theorem of R. Datko for stability in average. Syst. Control. Lett. 2016, 96, 1-6. [CrossRef]

11. Dragičević, D. Datko-Pazy conditions for nonuniform exponential stability. J. Differ. Equ. Appl. 2018, 24, 344-357. [CrossRef]

12. Sasu, B. Generalizations of a theorem of Rolewicz. Appl. Anal. 2005, 84, 1165-1172. [CrossRef]

13. Barreira, L.; Valls, C. Polynomial growth rates. Nonlinear Anal. 2009, 71, 5208-5219. [CrossRef]

14. Bento, A.J.G.; Silva, C. Stable manifolds for nonuniform polynomial dichotomies. J. Funct. Anal. 2009, 257, 122-148. [CrossRef]

15. Hai, P.V. On the polynomial stability of evolution families. Appl. Anal. 2015, 95, 1239-1255.

16. Barreira, L.; Fan, M.; Valls, C.; Jimin, Z. Robustness of nonuniform polynomial dichotomies for difference equations. Topol. Methods Nonlinear Anal. 2011, 37, 357-376.

17. Pinto, M. Perturbation of asymptotically stable differential systems. Analysis 1984, 4, 161-175. [CrossRef]

18. Boruga(Toma), R.; Borlea(Pătraşcu), D.I.; Toth, D.M-M. On uniform stability with growth rates in Banach spaces. In Proceedings of the 2021 IEEE 15th International Symposium on Applied Computational Intelligence and Informatics (SACI), Timisoara, Romania, 19-21 May 2021 ; pp. 393-396.

19. Mihiţ, C.L. On uniform h-Stability of evolution operators in Banach spaces. Theory Appl. Math. Comput. Sci. 2016, 6, 19-27. 